\title{
Propuestas para la mejora continua del área de producción de una empresa productora de carne de cerdo, mediante herramientas de manufactura esbelta
}

\section{Proposals for the continuous improvement of the production area of a pork production company, using lean manufacturing tools}

\author{
CONANT-PABLOS, Marco Antonio $\dagger^{*}$, FORNÉS-RIVERA, René Daniel, CANO-CARRASCO, \\ Adolfo y SÁNCHEZ-OSUNA, Adrián
}

Instituto Tecnológico de Sonora. Departamento de Ingeniería Industrial. Ciudad Obregón, Sonora. Colonia Villa ITSON. C.P. 85000

ID $1^{\mathrm{er}}$ Autor: Marco Antonio, Conant-Pablos / ORC ID: 0000-0002-3364-3702, Researcher ID Thomson: G-3911-2018, CVU CONACYT ID: 687331

ID $1^{\text {er }}$ Coautor: René Daniel, Fornés-Rivera / ORC ID: 0000-0002-7438-0056, Researcher ID Thomson: G-3906-2018, arXiv Author ID: rene_fornes, CVU CONACYT ID: 280435

ID $2^{\text {do }}$ Coautor: Adolfo, Cano-Carrasco / ORC ID: 0000-0002-3392-3667, Researcher ID Thomson: G-5035-2018, arXiv Author ID: Adolfo.cano, CVU CONACYT ID: 266064

ID $3^{\text {er }}$ Coautor: Adrián, Sánchez-Osuna / ORC ID: 0000-0001-8233-8918

DOI: $10.35429 / \mathrm{JTO} \cdot 2019.9 .3 .1 .14$

Recibido 10 de Enero, 2019, Aceptado, 30 de Marzo, 2019

Resumen

El presente proyecto tuvo lugar en una empresa productora de carne de cerdo, la cual desea disminuir los desperdicios de los productos del área de rebanado, específicamente en los productos Belly Slice, Kataroso Slice 120 gr y Belly Slice $(3 \mathrm{~cm})$, teniendo como objetivo identificar actividades que no agregan valor, a través de herramientas de manufactura esbelta, para proponer acciones de mejora en el proceso de rebanado en el área de producción. El procedimiento utilizado consistió en describir las actividades del proceso, generar tabla de especificaciones de los productos, determinar tiempo de ciclo y capacidad productiva, determinar tamaño de muestra, elaborar mapa de valor (VSM, por sus siglas en inglés) del proceso actual, identificar actividades que generan desperdicio, realizar VSM de la situación futura y, por último, generar propuestas de mejora. Como resultado, se obtuvo un programa para el monitoreo de la producción, adaptar la línea de producción a modo de celda de manufactura y la creación de un formato para el mantenimiento preventivo de la maquinaria y equipo; así como también la información que sentará las bases para los productos bajo estudio. Con dichas acciones se pretende aportar a la mejora continua de los indicadores de rendimiento, productividad y eficiencia.

Desperdicio, Manufactura esbelta, Mejora continua

\begin{abstract}
The present project took place in a pork production company, which wants to reduce the waste of the products of the slicing area, specifically in the products Belly Slice, Kataroso Slice $120 \mathrm{gr}$ and Belly Slice $(3 \mathrm{~cm})$, aiming to identify activities that do not add value, through lean manufacturing tools, to propose improvement actions in the slicing process in the production area. The procedure used was to describe the activities of the process, generate specifications table of products, determine cycle time and productive capacity, determine sample size, prepare Value Stream Map (VSM) of the current process, identify activities that generate waste, perform VSM of the future situation and, finally, generate proposals for improvement. As a result, was obtained a program to monitor production, adapt the production line as a manufacturing cell and create a format for preventive maintenance of machinery and equipment; as well as the information that will lay the foundations for the products under study. With these actions, the intention is to contribute to the continuous improvement of performance, productivity and efficiency indicators.
\end{abstract}

Waste, Lean manufacturing, Continuous improvement

Citación: CONANT-PABLOS, Marco Antonio, FORNÉS-RIVERA, René Daniel, CANO-CARRASCO, Adolfo y SÁNCHEZ-OSUNA, Adrián. Propuestas para la mejora continua del área de producción de una empresa productora de carne de cerdo, mediante herramientas de manufactura esbelta. Revista de Operaciones Tecnológicas. 2019. 3-9: 1-14

\footnotetext{
* Correspondencia del Autor (Correo electrónico: marco.conant@itson.edu.mx)

$\dagger$ Investigador contribuyendo como primer autor.
} 


\section{Introducción}

Debido al crecimiento económico que se experimentó durante el año 2018 en un contexto mundial, detonaron un aumento en la producción mundial de carne, permitiendo alcanzar un volumen de 335 millones de toneladas, lo que equivaldría a un crecimiento de $1.5 \%$ (5 millones de toneladas) con respecto al año 2017. Dejando, de acuerdo al informe realizado por la FAO en el mes de julio del año 2018, un consumo per cápita promedio de 43.9 kilogramos, es decir, un $0.6 \%$ más que en 2017 y se prevé que la carne de cerdo siga siendo la que más contribuya al aumento de la producción mundial de carne este año, seguida de la carne de aves de corral, de bovino y ovino (División de comercio y Mercados de la FAO, 2018).

Como refuerzo al argumento anterior, y con especial enfoque en la carne de cerdo, el Departamento de Agricultura de los Estados Unidos (USDA) estimó un crecimiento de $2.2 \%$ con respecto al año 2017, lo que permitiría alcanzar una producción mundial de 113.4 millones de toneladas. Éste mercado, el de la carne porcina, ha crecido significativamente durante las últimas décadas, incrementándose su consumo en más de un $80 \%$ en los últimos 30 años a nivel mundial (Sigaudo y Terré, 2018).

De acuerdo a Sigaudo y Terré (2018), el gran determinante de estos cambios es China, al ser el principal productor y consumidor a nivel mundial, cuya producción representa cerca del $50 \%$ del volumen total (54 millones ton). Por otra parte, la producción porcina de la Unión Europea (UE) representaría aproximadamente el $21 \%$ del total mundial (unos 24 millones de ton en el 2018), posicionándose como la segunda entidad con mayor producción de carne de cerdo. En tercer lugar, se encontraría Estados Unidos con una proyección del $11 \%$ del total mundial equivalente a 12 millones de ton y en cuarto lugar Brasil con 3.6 millones de toneladas (3\%). Juntos, los primeros cuatro productores de cerdo constituirían más del $80 \%$ de la producción mundial (Sigaudo y Terré, 2018).

En lo que respecta a las exportaciones de carne de cerdo, la Unión Europea (UE) participó con $35.7 \%$ de las exportaciones totales en el 2018 colocándose como principal exportador. En segundo y tercer lugar se encuentra Estados Unidos (EU) con $31.8 \%$ y Canadá con $15.8 \%$ respectivamente y por ultimo figura Brasil como la cuarta potencia exportadora.
Estos países en conjunto concentran el 91\% de las exportaciones mundiales (Gaucín, 2019). En contraparte, del lado de la demanda, los principales importadores son: China, Japón, México, Corea del Sur, Hong Kong y Estados Unidos, que concentran el $74 \%$ de las importaciones globales. China encabeza el listado como principal importador, superando desde 2017 al hasta entonces líder, Japón. Las importaciones chinas se duplicaron en los últimos 5 años, siendo la UE el principal proveedor de carne porcina de China, secundado por Canadá y Estados Unidos (Sigaudo y Terré, 2018).

El tercer mayor importador a nivel mundial es México, cuyas compras ascenderían al $14 \%$ del total en 2018 (1.2 millones de toneladas). Gracias al tamaño y dinamismo de su economía, México es un importador clave. En la última década, las compras de éste país crecieron a un ritmo promedio del $9 \%$ anual. (Sigaudo y Terré, 2018).

Entre los factores que explican el aumento en el consumo de carne de cerdo se encuentran los precios relativamente accesibles del cárnico, el aumento en el ingreso de los consumidores, así como la creciente confianza del consumidor, ya que, se cuenta con mayor información sobre las medidas de salubridad e inocuidad en las unidades de producción porcina. De ésta forma la percepción de que la carne de cerdo es una fuente de proteína saludable, similar a la carne de res y pollo ha crecido considerablemente (FIRA, 2016)

Aunado a lo anterior, el aumento del consumo de cerdos crece tanto en países con mercados ya consolidados, como en aquellos en vía de desarrollo. En América Latina el consumo porcino creció en los últimos años, especialmente en Colombia, México, Uruguay y algunos países de América Central; por lo que, dado que la demanda en la región crece a un ritmo mayor que la oferta interna, estas economías deben importar parte de lo que consumen a otros países productores. (Sigaudo y Terré, 2018).

Focalizándose en el mercado mexicano, actualmente la demanda interna de carne de cerdo excede la producción, lo que lleva a una fuerte dependencia de las importaciones extranjeras.

CONANT-PABLOS, Marco Antonio, FORNÉS-RIVERA, René Daniel, CANO-CARRASCO, Adolfo y SÁNCHEZ-OSUNA, Adrián. Propuestas para la mejora continua del área de producción de una empresa productora de carne de cerdo, mediante herramientas de manufactura esbelta. Revista de Operaciones Tecnológicas. 2019 
De acuerdo con cifras preliminares del SIAP (Servicio de Información Agroalimentaria y Pesquera) a diciembre del 2017, la Producción Nacional de carne de cerdo alcanzó 1'439,933 toneladas anuales, cantidad $4.6 \%$ mayor al observado en el 2016, con un valor estimado de 56,893 millones de pesos (Lobato, Gonzalez, y Teresa, 2018).

Port lo anterior, el gobierno de México detectó la oportunidad para llevar esta práctica a un nivel de mayor sofisticación y eficiencia, implementando programas de financiamiento gubernamental, los cuales tuvieron especial impacto en la región norte de México, en donde grandes empresas han conseguido refinar sus procesos para adentrarse en el mercado mundial (Hernández \& Parrish, 2017).

Dado que el gobierno del país ha tomado cartas en el asunto, las instalaciones inspeccionadas por el gobierno federal (conocidas como TIF o Tipo de Inspección Federal) continúan expandiéndose, puesto que en los últimos años SENASICA (Servicio Nacional de Sanidad, Inocuidad y Calidad Agroalimentaria) ha declarado que la tendencia a expandir la producción basada en TIF y reducir la producción municipal permitirá al sector garantizar la producción y el procesamiento de productos seguros para el consumo en el hogar y en el mercado de exportación (Hernández y Parrish, 2017).

En los últimos años, México ha trabajado de forma intensa para conquistar al mercado japonés y tenerlo en su cartera de clientes frecuentes. Cabe señalar que recientemente México recibió el reconocimiento por parte de Japón como productor libre de la Peste Porcina Clásica (PPC), lo que permitiría exportar carne de cerdo proveniente de todas las entidades federativas del país. Además, México continúa impulsando el desarrollo de otros mercados de exportación en Asia (FIRA, 2016).

En México, los seis principales estados productores de carne de cerdo son Jalisco, con 265 mil 217 toneladas; Sonora, 234 mil 639; Puebla, 166 mil 947; Yucatán, 135 mil 442; Veracruz, 122 mil 329, y Guanajuato, $110 \mathrm{mil}$ 489 toneladas. Se registró que el inventario nacional es de más de 1.7 millones de cabezas de ganado porcino y el consumo Per Cápita en México es de 16.3 kilogramos (SAGARPA, 2017).
Así pues, en palabras del subsecretario de Ganadería de la Secretaría de Agricultura, Ganadería, Recursos Hidráulicos, Pesca y Acuacultura (SAGARHPA), la porcicultura sonorense concentra el $17 \%$ de la carne de cerdo en México, ocupando el segundo lugar en la producción nacional y generando más de 19 mil empleos. Además, subrayó que durante 2019 se contempla incrementar las exportaciones debido a la gran aceptación y calidad de esta carne en los mercados internacionales; logros alcanzados por los productores sonorenses en base a las buenas prácticas de producción (Redacción Entorno, 2018).

Es importante destacar que en 2016 cinco establecimientos Tipo Inspección Federal (TIF) pertenecientes a la UGRPS (Unión Ganadera Regional de Porcicultores de Sonora), exportaron 67 mil 419 toneladas de carne de cerdo a países como, Japón, China, Corea y Estados Unidos, con valor superior a los 272 millones de dólares, ocupando el primer lugar nacional con el $66 \%$ de las exportaciones. A términos del año 2017 se incrementó la cantidad de producto exportado a más de 80 mil toneladas de carne de cerdo con valor superior a los 300 millones de dólares (Gobierno del estado de Sonora, 2018) Es así que la Unión Ganadera Regional de Porcicultores de Sonora, agregó el Subsecretario de Ganadería de la SAGARHPA, está conformada por cuatro asociaciones ganaderas locales (Hermosillo, Cajeme, Navojoa y Huatabampo), y cuenta con 335 granjas y 148 mil 674 vientres. (Redacción Entorno, 2018).

La Unión Ganadera Regional de Porcicultores de Sonora tiene su sede en $\mathrm{Cd}$. Obregón; de esta forma en el municipio de Cajeme se encuentran algunas de las granjas exportadoras más importantes del país, las cuales cuentan con todo un complejo de crianza y procesamiento para éste insumo primario. Con una producción estimada de un millón 200 mil cerdos al año, la región del Valle del Yaqui es la que más destina cerdo hacia el mercado de exportación (Monteverde, 2018). Sin embargo, de acuerdo al presidente de la Asociación Ganadera Local de Porcicultores de Cajeme (AGLPC), a pesar de que los índices de producción local han ido en aumento, las importaciones estadounidenses no han parado, al contrario, éstas van en aumento, lo que afecta seriamente a los productores de la región, los cuales no pueden competir con los precios ofrecidos por el vecino del norte. (Redacción, 2018).

CONANT-PABLOS, Marco Antonio, FORNÉS-RIVERA, René Daniel, CANO-CARRASCO, Adolfo y SÁNCHEZ-OSUNA, Adrián. Propuestas para la mejora continua del área de producción de una empresa productora de carne de cerdo, mediante herramientas de manufactura esbelta. Revista de Operaciones Tecnológicas. 2019 
Para lograr lo anterior, las empresas productoras de carne de cerdo de la región deben ser capaces de satisfacer a su mercado objetivo con la suficiente eficiencia, de tal forma que les permita ser competitivos en precio. En pocas palabras, las empresas se verán beneficiadas a partir de unos procesos de negocio eficientes. (Gisbert, 2018).

Además de los factores externos como los valores internacionales de la carne, que afectan la competitividad de los productores locales, existen otros factores, los cuales son de carácter interno, por lo que están al alcance de las organizaciones para ser controlados. Uno de los principales es el desperdicio, que, de acuerdo a los autores Jiménez y Gisbert (2017), la palabra desperdicio está ligada con aquellas actividades realizadas en el proceso productivo que hace uso de recursos como materia prima, tiempo, equipo, materiales y personas, pero que no agregan valor al producto, por lo que, en resumidas cuentas, es todo aquello por lo que el cliente no está dispuesto a pagar.

Mientras que para Giannasi (2012), desperdicio es: "Todo aquello que no agrega valor a un producto o servicio para sus clientes, por lo que es toda mal utilización de los recursos y/o posibilidades de la empresa". Egas (2017), define desperdicio como: "El desprendimiento de material que no representa valor agregado al cliente y que no puede ser aprovechado en la generación del mismo, pero que, en sí, es necesario para generar el producto"

Este factor tiene tanto efecto en la eficiencia, el rendimiento, como en la productividad; los cuales son definidos, en el caso de la eficiencia, como aquella comparación entre los valores observados y los valores óptimos, tanto para sus entradas como para sus salidas, haciendo énfasis en su grado de aprovechamiento, por lo que es de vital importancia una constante vigilancia (Álvarez, 2014). El rendimiento sería la proporción entre el resultado que se obtiene y los medios que se emplearon para alcanzar al mismo. (Quezada, 2016). Y la productividad, otro concepto que complementa al de desperdicio, es la cantidad de recursos utilizados y la cantidad de bienes y servicios producidos. Por ende, la productividad es un índice que relaciona lo producido por un sistema (salidas o producto) y los recursos utilizados para generarlo (entradas o insumos). (Carro y Gonzalez, 2012).
Gracias al fuerte impacto que tiene este mercado en el municipio de Cajeme, el presente proyecto se enfocará en una de las principales empresas porcícolas de la región, en donde se analizarán a detalle sus procesos productivos, misma que se describe a continuación. La planta tiene una capacidad para procesar 1250 cerdos diarios y los productos que genera se exportan hacia el mercado de Japón, Corea, Estados Unidos y algunos países europeos. La empresa atiende su demanda nacional mediante sucursales en México, Acapulco y Cancún que operan mayoreo hacia: Yucatán, Campeche, Tabasco, Puebla, Veracruz, Oaxaca, Guerrero, Estado de México y Morelos. En cuanto a su demanda regional cuenta con una oficina matriz en Cd. Obregón, cubriendo el noroeste del país (Empresa, 2019b).

De esta forma la compañía pertenece a aquellas empresas que cuentan con la calidad e higiene suficiente de acuerdo a lo establecido, con el claro objetivo de brindar a los consumidores un producto en condiciones favorables. La empresa posee una Planta de Rastro TIF, que divide su infraestructura en la estancia de corrales, sacrificio, corte, valor agregado y embarques.

En la planta existen distintas áreas, en donde cada una abastece a otra. En el área de sacrificio se reciben los cerdos que cumplen con todas las características necesarias para ser sacrificados y que son criados en granjas de la organización. En ésta área se sacrifican los cerdos, posteriormente pasan al área de corte, se clasifican y se canalizan a su área correspondiente. Bajo esta perspectiva y a grandes rasgos las áreas más importantes de la empresa son las siguientes: el área de sacrificio, el área de corte y el área de valor agregado. En ésta última se dispone de trabajos mucho más elaborados y detallados que los encontrados en el área de corte o sacrificio.

De acuerdo a Gisbert (2018), al definir cada proceso de la empresa, se deben formular aquellos indicadores que servirán al responsable para controlarlo, así como para informar del desempeño del proceso a otros miembros de la organización. 
Por lo que, aunque la mayoría de las actividades realizadas en el objeto bajo estudio presentan un alto grado de mecanización, éstas se deben monitorear constantemente con ayuda de parámetros tan críticos como lo es el desperdicio, la eficiencia, la productividad y el rendimiento, que de acuerdo a entrevistas con los directivos del departamento de producción, la asociación de éstos indicadores permite conocer si los productos obtenidos por sus procesos están siendo elaborados de modo que se cumplan con los niveles mínimos de desperdicio, que para la situación bajo estudio, varían de un producto a otro (Empresa , 2019a).

A pesar de que lo que se obtiene como "desperdicio" es aprovechado para la elaboración de subproductos, actualmente la empresa tiene establecido como meta que, para el producto Belly Slice $2.5 \mathrm{~mm}$ el porcentaje de desperdicio permisible no debe de sobre pasar el $17.6 \%$ de la materia prima introducida, para el producto Kataroso Slice $120 \mathrm{gr}$, la cifra se reduce hasta el $12.32 \%$ y por último se encuentra el producto Belly Slice $3 \mathrm{~cm}$, el cual tiene como nivel máximo de desperdicio un 11\% (Empresa , 2019b).

Debido a la introducción de productos nuevos en la línea de rebanado, se requieren establecer los estándares para aquellos indicadores asociados con el control de desperdicios (el rendimiento, la productividad y eficiencia), que se describen con detalle a continuación:

El rendimiento, que varía para cada producto y que se obtiene a partir del porcentaje de materia prima que se convirtió en producto terminado, está representando un obstáculo para la organización, ya que debido a la introducción de estos nuevos productos (Belly Slice, Belly Slice $(3 \mathrm{~cm})$ y Kataroso Slice 120gr), se desconoce dicho parámetro y por consecuente el estándar al que debe estar trabajando cada uno de ellos.

El segundo indicador de interés es la productividad, que se obtiene al contabilizar la cantidad de producto terminado (en $\mathrm{kg}$ ) obtenidos por operario.
El tercer parámetro en cuestión es la eficiencia, que no es más que la comparación del rendimiento obtenido contra el estándar establecidos (Empresa, 2019a).

A partir de la presente situación en la que se desconocen los parámetros más críticos como lo son el desperdicio, rendimiento, eficiencia y productividad, de aquellos productos de nueva introducción en la línea de rebanado, la empresa bajo estudio requiere de una intervención para la realización de un análisis determinístico que permita identificar aquellas acciones que generan desperdicios en el proceso, en virtud de un óptimo aprovechamiento de sus recursos tanto financieros, como materiales, tecnológicos $\mathrm{y}$ humanos, que presentan margen de mejora en materia de desperdicio, como lo evidencia la información obtenida del proceso mostrada a continuación (Empresa , 2019a).

A continuación, en la tabla 1, se muestra los indicadores con los que está trabajando cada uno de los productos bajo estudio (ver Tabla 1).

\begin{tabular}{|c|c|c|c|}
\hline Producto & $\begin{array}{c}\text { Belly } \\
\text { Slice } \\
2.5 \\
\mathrm{~mm}\end{array}$ & $\begin{array}{l}\text { Belly } \\
\text { Slice } \\
3 \mathrm{~cm}\end{array}$ & $\begin{array}{c}\text { Kataroso } \\
\text { Slice } 120 \\
\text { gr }\end{array}$ \\
\hline Productividad (Kg/Operario) & 70 & 75 & 60 \\
\hline Subproductos (\%) & 27.6 & 12.18 & 23.22 \\
\hline Desperdicio $(\mathrm{Kg})$ & 1186 & 352 & 2861 \\
\hline Merma (\%) & 0.82 & 1.02 & 1.4 \\
\hline Rendimiento $(\%)$ & 71.2 & 86.8 & 74.9 \\
\hline Rendimiento Estándar & 82.4 & 89 & 87.68 \\
\hline Eficiencia (\%) & 86.4 & 97.5 & 85.4 \\
\hline
\end{tabular}

Tabla 1 Eficiencia de los Productos

Fuente: Departamento de Producción (Empresa, 2019b)

En la Tabla 1, se puede observar una brecha considerable en el indicador de eficiencia del proceso de rebanado en los productos de interés, especialmente en los productos Belly Slice $2.5 \mathrm{~mm}$ y Katoroso Slice $120 \mathrm{gr}$, los cuales distan $13.6 \%$ y $14.58 \%$ respectivamente del objetivo que es alcanzar en su totalidad el porcentaje de rendimiento establecido y con ello conseguir una eficiencia del $100 \%$. Además, se debe poner un especial cuidado en el producto Kataroso Slice 120 gr, ya que es un producto realizado con materia prima importada de Canadá, lo que impacta fuertemente su desperdicio en los costos de producción (Empresa, 2019b). 
Una vez expuesta la situación en la que se encuentra el área de producción y siendo más específicos, el área de rebanado, dado que los indicadores de rendimiento, productividad y eficiencia se encuentran por debajo de los niveles deseados, es necesario plantear acciones de mejora que permitan el aumento de dichos indicadores, buscando impactar positivamente en la disminución de los desperdicios generados por los productos introducidos.

\section{Planteamiento del Problema}

Debido a la introducción de nuevos productos en el proceso de rebanado, la empresa bajo estudio desconoce si los productos Belly Slice, Kataroso Slice $120 \mathrm{gr}$ y Belly Slice $(3 \mathrm{~cm})$ son elaborados de manera que se aprovechen al máximo los recursos disponibles; haciendo necesario plantear acciones de mejora que permitan el aumento de los indicadores de rendimiento, productividad y eficiencia.

\section{Objetivo}

Identificar actividades que no agregan valor, a través de la herramienta VSM, para proponer acciones de mejora en el proceso de rebanado en el área de producción.

\section{Revisión de Literatura}

Proceso productivo es la creación de un producto, el cual puede ser un bien o servicio mediante la combinación de factores necesarios para conseguir satisfacer la demanda del mercado mediante la utilización de elementos fundamentales como: el material, el recurso humano, la tecnología y el capital (Montoyo, 2011). Este proceso busca añadir valor y solo lo hace durante el tiempo en el que modifica la forma o las propiedades del producto para lograr los requisitos que el cliente valora. De forma puntual, valor agregado es todo aquello por lo que el cliente está dispuesto a pagar (Madariaga, 2013).

El producto no se ve reducido sólo al objeto físico, sino que es un completo conjunto de beneficios o satisfacciones que los consumidores perciben que obtienen cuando lo adquieren (Abdú, 2013), y de acuerdo a Suñé (2010) puede ser tangible (producto) o intangible (servicio).
Así es que de acuerdo a Madariaga (2013), todo aquello, que como parte o consecuencia del proceso productivo que no agrega valor para el proceso o producto y por lo que el cliente no está dispuesto a pagar, se considera desperdicio, y a decir de Corredor (2015), estos se clasifican en siete diferentes tipos (sobreproducción, espera, transportes, movimientos, defectos, sobre procesamiento e Inventario).

De acuerdo a Hernández y Vizán (2013), el modelo de fabricación esbelta, conocido como Lean Manufacturing es una alternativa consolidada, por lo que su aplicación debe ser considerada por aquellas empresas que quieran desarrollar su mayor potencial, y consiste en la aplicación sistemática y habitual de un conjunto de técnicas de fabricación que buscan la mejora de los procesos productivos a través de la reducción de todo tipo de desperdicios. En cambio, Rojas y Gisbert (2017), la definen como aquella filosofía que trabaja bajo el enfoque de la mejora continua y optimización de un sistema de producción o de servicio, mediante la eliminación o disminución de desperdicio de todo tipo ya sea inventarios, tiempo, productos defectuosos, transportes, retrabajos o toda acción que no agregue valor al producto y por lo que el cliente no está dispuesto a pagar.

Sarria, Fonseca, y Bocanegra (2017), también la definen como una filosofía, pero agregan que cuenta como una metodología que consta de herramientas para el diagnóstico, la operatividad y seguimiento de áreas de oportunidad que propician el desarrollo de actividades o circunstancias que no agregan valor para el producto, proceso o para la empresa en general.

Lean Manufacturing consta de una "caja de herramientas" compuesta por: 5'S, Mantenimiento Productivo Total (TPM), SMED, Kan-ban, Value Stream Mapping (VSM). Esta última también conocida en Latinoamérica como mapeo de flujo de valor, la cual es un modelo gráfico que mediante símbolos específicos representa la cadena de valor, mostrando tanto el flujo de materiales como el flujo de información desde el proveedor hasta el cliente. 
Tiene por objetivo plasmar en un papel, de una manera sencilla, todas las actividades productivas para identificar la cadena de valor y detectar, a nivel global, donde se producen los mayores desperdicios del proceso (Hernández y Vizán, 2013), y la cual, de acuerdo a Benítez (2012), habitualmente se enfoca en la mejora del flujo de los materiales y actividades a lo largo de todo el proceso productivo.

\section{Método}

El objeto bajo estudio fue el área de rebanado en el departamento de producción del área de valor agregado y los materiales fueron: Cronómetro digital para la determinación de los tiempos de las actividades, báscula para el pesaje de la materia prima cuando era necesario determinar los rendimientos para cada producto, bitácora utilizada para registrar las lecturas generadas en la toma de tiempos y rendimientos, termómetro usado para verificar las condiciones de temperatura en las materias primas, y tabla de porcentajes para asignar márgenes o tolerancias editada por la Oficina Internacional del Trabajo (OIT).

El procedimiento utilizado se basó en Socconini (2017), Ruíz (2016), Sánchez y Baldenegro (2018), y consistió en lo siguiente:

1) Describir las actividades del proceso: Como medio para la descripción del proceso de rebanado, se elaboró un diagrama SIPOC detallando los recursos necesarios y las métricas de control pertinentes.

2) Generar tabla de especificaciones de los productos: Estas fueron proporcionadas por el jefe del departamento de producción y se elaboró una tabla con las especificaciones de los productos.

3) Calcular tiempo de ciclo de las actividades: Para la determinación del tiempo de ciclo de elaboración de cada uno de los productos se sondeó cada una de las actividades que conforman el proceso. Se cronometraron un total de tres actividades, el rebanado, realizado por la máquina de rebanado y las actividades de pesaje y embolsado, realizadas por operarios. Para cada una de las actividades se tomaron un total de 20 lecturas, que son las que requiere el departamento de producción. Para el caso del rebanado de la materia prima, se tomó el tiempo que le toma a la rebanadora procesar la materia prima.
En cuanto a los tiempos de pesaje y embolsado, debido a que son actividades realizadas por operarios, se consultó con el encargado de línea sobre quien era apto para la realización de un muestreo, el cual debía ser aquel operario que demostraba mantener tiempos constantes, ni muy rápidos para trabajar por encima de la demanda, ni muy lentos como para no cumplirla, con el fin de que los tiempos obtenidos fueran equilibrados y alcanzables por todos los operarios en general.

El tiempo de ciclo se obtuvo del promedio de las lecturas generadas en los operarios sondeados, aplicando sus respectivas tolerancias conforme lo indica la tabla editada por la OIT, y la siguiente formula:

Ajuste por tolerancias $=\frac{100}{100-\sum \text { Tolerancias }}$

Con la finalidad de recopilar información concerniente a los tiempos de las actividades implicadas en la realización de los productos, así como también para obtener información relacionada con el producto en proceso, se determinó el tamaño de muestra que representa al total de la población, con ayuda de la siguiente fórmula:

$N_{i}=\left(\frac{z s_{i}}{E \bar{X}_{i j}}\right)^{2}$

Donde:

$\mathrm{S}_{\mathrm{i}}=$ Desviación estándar de la serie de desviaciones para el elemento de trabajo $i$.

$X_{i j}=$ Tiempo registrado para cada elemento de trabajo i, en la observación j.

$\mathrm{N}_{\mathrm{i}}=$ Número de observaciones requeridas.

$\mathrm{Z}=$ Calificación $\mathrm{Z}$ correspondiente al nivel deseado de confiabilidad

$E=$ Error permisible (5\%) (Freivalds y Niebel, 2014).

Dicha fórmula se aplicó para cada uno de los productos bajo estudio, para la determinación del tamaño de muestra idóneo que representara al total de la población y con ello obtener información verás del proceso.

4) Construir VSM actual: Para construir el VSM actual primero se dibujaron los íconos del cliente, proveedor y producción, los cuales se colocan en la parte superior del formato. 
Del lado derecho del formato se coloca el recuadro correspondiente al cliente y debajo de él se ingresan los requisitos correspondientes a la demanda de los productos bajo estudio, cuidando tener uniformidad en los valores, ya sean cajas o unidades demandadas. En el centro del formato se coloca el ícono correspondiente al área de producción y del lado izquierdo del formato se colocan a los proveedores del proceso, luego se dibujaron los íconos de entradas del proceso, luego, debajo del recuadro correspondiente a los clientes, se dibuja el ícono de las entradas, que son los insumos necesarios para que el proceso funcione, se agrega información de utilidad como lo son la cantidad y la frecuencia con la que se reciben los insumos, se colocan cada una de las actividades $u$ operaciones que componen al proceso, agregando información de relevancia como:

Tiempo de ciclo de la actividad, disponibilidad del equipo, número de operarios y el tiempo total disponible para la realización de dicha actividad, y f Finalmente se añade una línea de tiempo en la parte inferior de la hoja, en donde se coloca el tiempo muerto generado por los inventarios a lo largo del proceso, así como también el tiempo de procesamiento o de valor agregado para el proceso de rebanado.

5) Identificar actividades que generan desperdicio o no agregan valor: Se identificaron todas aquellas oportunidades de mejora que estaban generando desperdicio mediante análisis de parte de todos los involucrados en el proceso de mejora y se resaltaron en el VSM actual con ayuda de un estallido kaizen.

6) Realizar VSM de la situación futura: Para la realización del mapa de flujo de valor futuro se consideraron todas las áreas de oportunidad identificadas en el mapeo actual y con ello se diseñó el proceso que busca combatir la proliferación de desperdicios en cualquiera de las actividades que lo componen, por lo que se plasmó en el VSM de la situación futura la forma en cómo debe cambiar el proceso para alcanzar niveles de eficiencia más elevados, considerando los aspectos críticos que habían sido encerrados por un estallido kaizen, plasmando los valores ideales para la organización, los cuales se busca alcanzar con las propuestas de mejora.
7) Generar propuestas de mejora: Se presentaron las mejoras que ayudarán a alcanzar la situación plasmada en el mapeo de flujo de valor futuro, con las cuales se busca atender a aquellas áreas que presentan desperdicios y que fueron identificadas con un estallido kaizen.

\section{Resultados}

1) En el diagrama SIPOC que se muestra en la figura 1 , se describe con detalle cada uno de los factores que interactúan en el proceso productivo (ver Figura 1).



Figura 1 Sipoc del proceso de rebanado

Fuente: Elaboración propia con información del departamento de producción del área de valor agregado

El diagrama SIPOC, presentado en la tabla 2, muestra la interacción que existe entre cada una de las actividades, así como los aspectos críticos a cuidar en sus entradas y salidas. 2) A continuación en la tabla 2, se muestran las especificaciones de los productos bajo estudio (ver Tabla 2). 


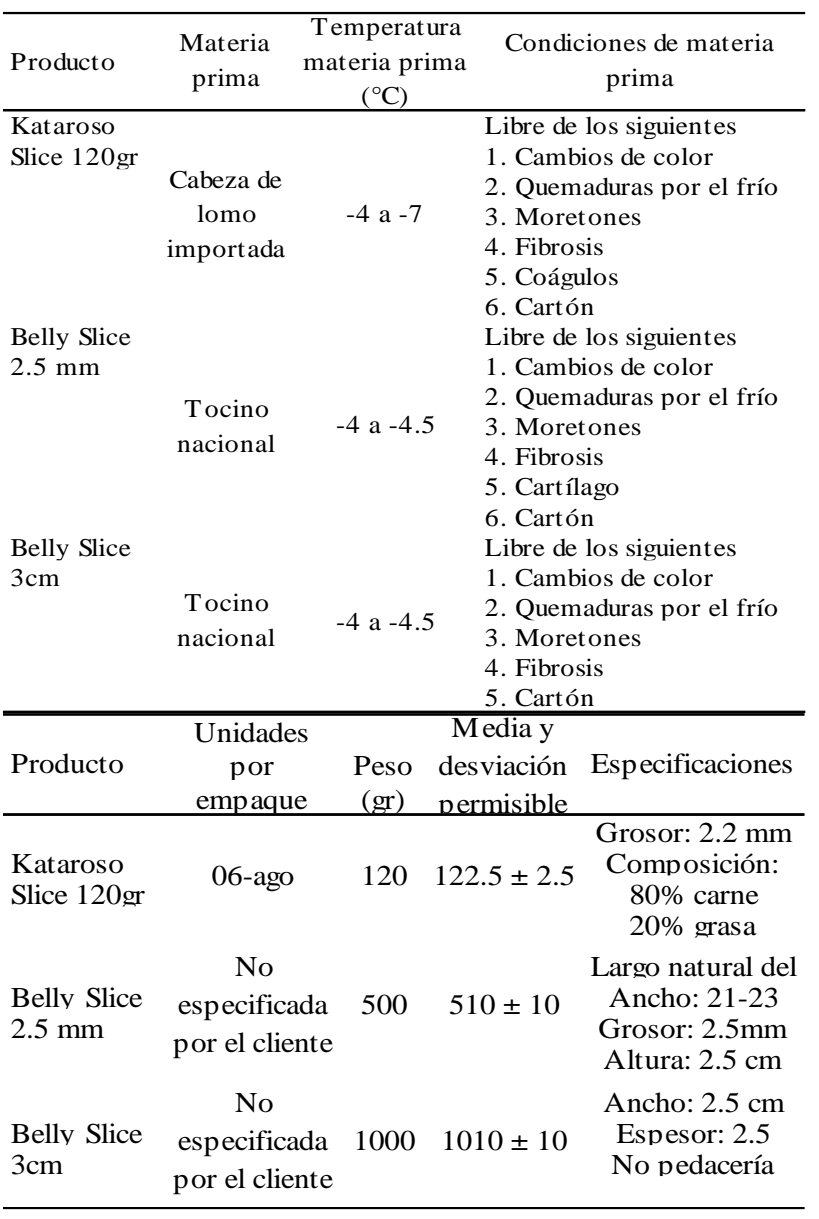

Tabla 2 Especificaciones de los productos

Fuente: Elaboración propia con información del departamento de producción del área de valor agregado

De acuerdo con el contenido de la tabla 2 , los productos bajo estudio comparten una serie de características que deben de cumplir para ser aptos para su procesamiento, así como otras que son muy particulares a cada uno de los productos.

3) A continuación, en la tabla 3, se presenta el tamaño de la muestra.

\begin{tabular}{|l|r|}
\multicolumn{1}{|c}{ Productos } & $\begin{array}{c}\text { Número de observaciones } \\
\text { a cronometrar }\end{array}$ \\
\hline Kataroso Slice $120 \mathrm{gr}$ & 198 \\
\hline Belly Slice $2.5 \mathrm{~mm}$ & 138 \\
\hline Belly Slice $3 \mathrm{~cm}$ & 111 \\
\hline
\end{tabular}

Tabla 3 Número de observaciones para estudio de tiempo Fuente: Elaboración Propia

Una vez obtenidas todas las observaciones requeridas se calculó el tiempo de ciclo considerando las tolerancias constantes (9\%) y variables (2\% por estar de pie y $4 \%$ por condiciones de baja temperatura), además de un $1 \%$ adicional, para el caso de congelado y enfundado de Belly Slice $2.5 \mathrm{~mm}$. (ver Tabla 4).

\begin{tabular}{|c|c|c|c|c|}
\hline Producto & Actividad & $\begin{array}{l}\text { Tiempo } \\
\text { promedio } \\
\text { (segs.) }\end{array}$ & $\begin{array}{l}\text { Tolerancias } \\
\text { (pts.) }\end{array}$ & $\begin{array}{l}\text { Tiempo de } \\
\text { ciclo (segs.) }\end{array}$ \\
\hline Todos & $\begin{array}{l}\text { Congelado } \\
\text { (transporte) }\end{array}$ & 133 & 16 & 158 \\
\hline \multirow{5}{*}{$\begin{array}{l}\text { Belly } \\
\text { Slice } 3 \mathrm{~cm}\end{array}$} & $\begin{array}{l}\text { Corte en } \\
\text { sierra }\end{array}$ & 17 & 15 & 20 \\
\hline & $\begin{array}{l}\text { Acomodo } \\
\text { en } \\
\text { rebanadora }\end{array}$ & 80.75 & 15 & 95 \\
\hline & Rebanado & 38.55 & 15 & 38.55 \\
\hline & Pesaje & 12.19 & 15 & 14.34 \\
\hline & Embolsado & 101.85 & 15 & 120 \\
\hline \multirow{3}{*}{$\begin{array}{l}\text { Belly } \\
\text { Slice } 2.5 \\
\mathrm{~mm}\end{array}$} & Rebanado & 25 & 15 & 25 \\
\hline & Pesaje & 36.41 & 15 & 43 \\
\hline & Embolsado & 45.4 & 15 & 53.41 \\
\hline \multirow{4}{*}{$\begin{array}{l}\text { Kataroso } \\
\text { Slice } 120 \\
\text { gr }\end{array}$} & Enfundado & 63.69 & 16 & 75 \\
\hline & Rebanado & 55.45 & 15 & 55.45 \\
\hline & Pesaje & 9.95 & 15 & 11.7 \\
\hline & Embolsado & 12.31 & 15 & 14.5 \\
\hline $\begin{array}{l}\text { Kataroso } \\
\text { y Belly } \\
2.5 \mathrm{~mm} \\
\end{array}$ & $\begin{array}{l}\text { Empacado } \\
\text { al vacío }\end{array}$ & 11 & 15 & $\begin{array}{l}13+20(\text { tiempo } \\
\text { máquina })=33\end{array}$ \\
\hline
\end{tabular}

Tabla 4 Tiempos de ciclo de las actividades del proceso de rebanad

Fuente: Elaboración Propia

4) Con la información anterior se dibujó el VSM actual, quedando como se muestra a continuación en la figura 2 (ver Figura 2).

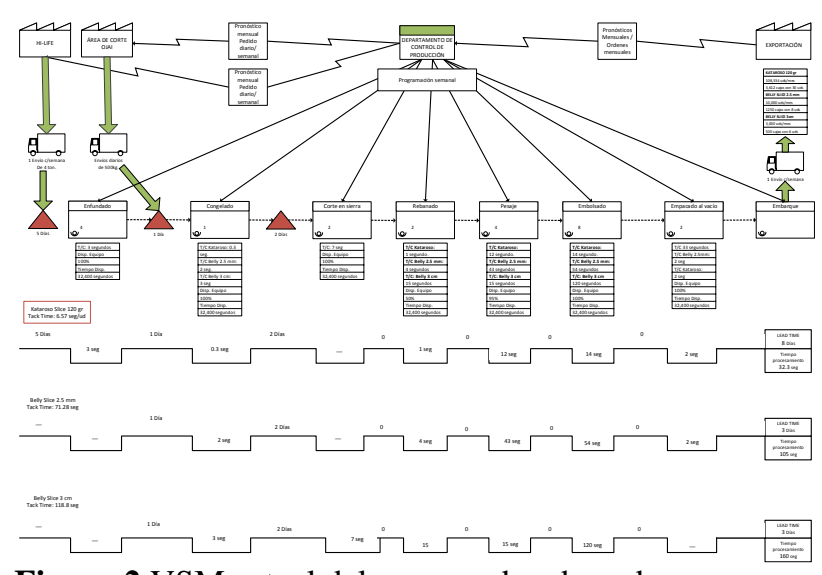

Figura 2 VSM actual del proceso de rebanado

Fuente: Elaboración Propia

En la figura 2 se puede observar, además del flujo de los materiales y la información a través de las actividades del proceso productivo de rebanado, datos improtantes de la actividad, como el tiempo de ciclo, el número de operarios, la disponibilidad del equipo y el tiempo disponible.

5) A continuación, en la figura 3 , se muestra el VSM con la identificación de aquellas actividades que generan algún tipo de desperdicio para el proceso de rebanado (ver Figura 3). 


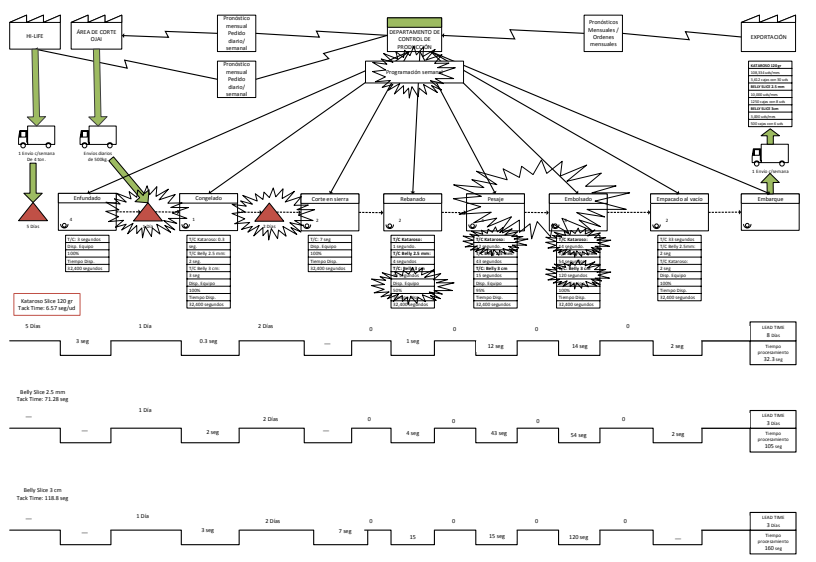

Figura 1 VSM con identificación de desperdicios Fuente: Elaboración Propia

En la figura 3, se logra observar una serie de actividades encerradas en un estallido kaizen, las cuales no se apegan a la filosofía Lean, bajo la cual trabaja la herramienta de VSM, por lo que su identificación ayuda para dirigir los esfuerzos hacia aquellas actividades que requieren de medidas correctivas, y con ello dirigirse hacia el camino de la mejora continua.

6) En la tabla 5, se enlistan las propuestas de mejora para la solución de las fuentes de desperdicio identificadas previamente (ver Tabla 5).

\begin{tabular}{|c|c|c|c|}
\hline $\begin{array}{c}\text { Mejora } \\
\text { propuesta }\end{array}$ & $\begin{array}{c}\text { Área de } \\
\text { aplicación }\end{array}$ & $\begin{array}{c}\text { Actividad } \\
\text { involucrada }\end{array}$ & $\begin{array}{l}\text { Indicador al } \\
\text { que impacta }\end{array}$ \\
\hline $\begin{array}{l}\text { Programa para } \\
\text { el monitoreo } \\
\text { de } \\
\text { producción la }\end{array}$ & $\begin{array}{l}\text { Oficina de } \\
\text { producción }\end{array}$ & $\begin{array}{l}\text { Planeación de } \\
\text { la producción }\end{array}$ & $\begin{array}{l}\text { Rendimiento, } \\
\text { Productividad } \\
\text { y Eficiencia }\end{array}$ \\
\hline $\begin{array}{l}\text { Celda de } \\
\text { manufactura }\end{array}$ & \begin{tabular}{|ll}
$\begin{array}{l}\text { Área } \\
\text { rebanado }\end{array}$ & de \\
\end{tabular} & $\begin{array}{ll}\begin{array}{l}\text { Pesaje } \\
\text { embolsado }\end{array} & \text { y } \\
\end{array}$ & Produ \\
\hline $\begin{array}{l}\text { Formato para } \\
\text { mantenimiento } \\
\text { preventivo }\end{array}$ & $\begin{array}{l}\text { Mantenimiento } \\
\text { del Área de } \\
\text { rebanado }\end{array}$ & \begin{tabular}{lr}
\multicolumn{2}{l}{ Mantenimiento } \\
de & la \\
maquinaria & $y$ \\
equipo & de \\
rebanado &
\end{tabular} & Productividad \\
\hline $\begin{array}{l}\text { Determinación } \\
\text { del tamaño de } \\
\text { la muestra }\end{array}$ & $\begin{array}{l}\text { Oficina de } \\
\text { producción }\end{array}$ & $\begin{array}{lr}\text { Muestreo } & \text { de } \\
\text { productos } & y \\
\text { operarios } & \\
\end{array}$ & Rendimiento \\
\hline
\end{tabular}

Tabla 5 Propuestas de mejora Fuente: Elaboración Propia

En la Figura 4, se ilustra el VSM de la situación futura, en donde se aplicaron las mejoras descritas en la Tabla 6 (ver Figura 4).

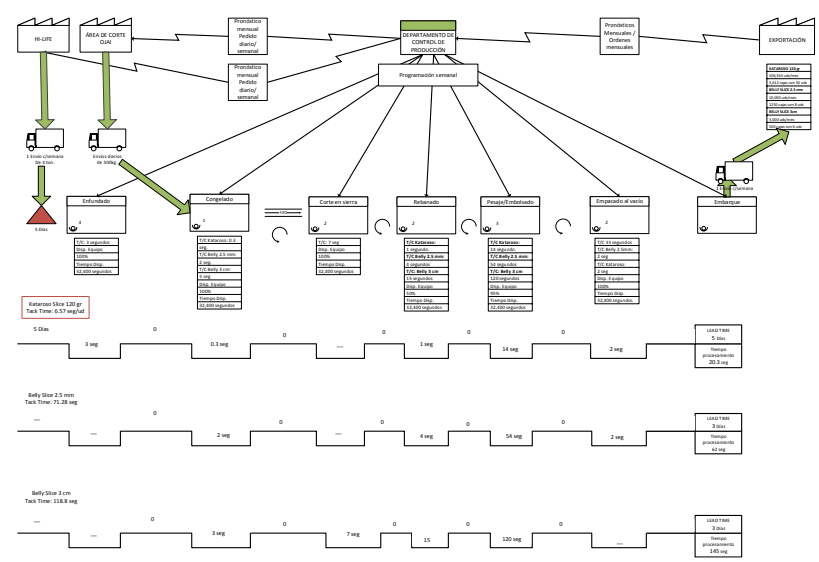

Figura 4 VSM futuro

Fuente: Elaboración Propia

En el VSM Futuro mostrado en la figura 4, se plasmaron las acciones de mejora propuestas, para la eliminación de las fuentes de desperdicio identificadas en la Figura 2.

7) A continuación se detallan las propuestas de mejora desarrolladas para combatir los desperdicios previamente identificados:

a) Programa para el monitoreo de la producción. Como parte de las propuestas de mejora, se diseñó una programa de monitoreo en Microsoft Excel, la cual lleva por nombre "Registro". En ella, en la primera hoja se muestra una interfaz, la cual dispone de los apartados de: Día (en donde se elaboró una lista con los días que labora el área), Fecha, Producto ( en donde se elaboró una lista con todos los productos que produce el área), Materia prima ( en donde se captura la cantidad de materia prima utilizada).

Producto terminado (donde se captura la cantidad total de producto terminado obtenido), Subproducto (nombre del subproducto obtenido), Cantidad de subproducto, Merma (en donde se elaboró una formula que resta la cantidad de producto terminado y de subproducto a la materia prima, para obtener con ello la merma), Operadores (donde se captura el número de operadores involucrados en el proceso), Rendimiento (donde se realizó una formula que determina el porcentaje de materia prima que se convirtió en producto terminado) y Productividad (división entre producto terminado y operadores). 
En la segunda hoja, correspondiente a la hoja de "Resumen", la interfaz muestra un concentrado de la información disponible en el programa, En la interfaz del lado izquierdo se dispone de una lista a modo de menú que contiene todos los productos del departamento, e inmediatamente debajo de ella se encuentra una tabla que concentra un acumulado de los siguientes criterios: Materia prima, producción total, operadores, rendimiento, productividad, y merma; así como tambien un indicador que muestra si se cumple o no con los indicadores de rendimiento y productividad, ilustrando también el margen de oportunidad a mejorar. La información de las tablas localizadas debajo de la lista de productos se actualiza de acuerdo al producto que se haya seleccionado.

Por último, la hoja tres, contiene la interfaz correspondiente al programa de monitoreo, que lleva por nombre "Datos", se realizó una tabla dinámica con los siguientes encabezados: día, fecha, producto, materia prima $(\mathrm{kg})$, producto terminado $(\mathrm{kg})$, subproducto 1 , cantidad subproducto 1 , subproducto 2 , cantidad subproducto 2, subproducto 3, cantidad subproducto 3, merma, operadores, rendimiento y productividad. Es en esta hoja a donde se dirigen los datos capturados en la hoja de registro, inmediatamente despues de que se presiona el botón "Guardar" el cual contiene un macro de registro.

b) Celda de trabajo. Atendiendo al estallido kaizen localizado en las actividades de pesaje y embolsado, en donde se detectó una sobrecarga de trabajo en los primeros cuatro embolsadores, como acción de mejora se propuso una celda de manufactura para tratar de balancear las cargas de trabajo en cada uno de los operarios, como se muestra a continuación (ver Figura 5).

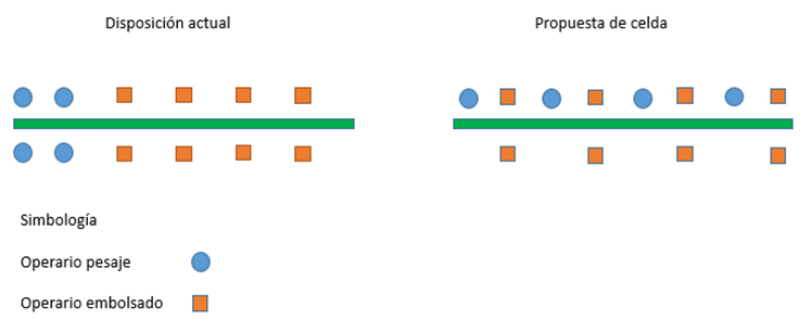

Figura 5 Propuesta de celda de manufactura. Fuente: Elaboración Propia
En la figura 5 se puede observar la distribución actual de la línea de rebanado, en donde ésta se encuentra dividida en dos secciones, la de pesaje y la de embolsado. En la situación actual persiste un desbalanceo en la carga de trabajo entre los operarios de embolsado, sobrecargando a la primera mitad de los operarios y presentando tiempo de ocio en la última mitad. En la propuesta de mejora los operarios se dispusieron a modo de celda de manufactura, en donde un pesador abastece a dos operarios de embolsado, repitiéndose la secuencia a lo largo de toda la banda transportadora, y con ello eliminando los tiempos de ocio y sobrecarga presentes en el escenario actual.

c) Formato para el mantenimiento preventivo. Como propuesta de solución a la problemática identificada en la disponibilidad de la maquinaria y equipo, en donde se cuenta con un porcentaje de $50 \%$, muy por debajo de los requerimientos de la organización, se diseñó un formato para el mantenimiento preventivo, donde se establece la periodicidad con la que se realiza el mantenimiento, el tipo de actividad realizada, así como las observaciones encontradas a lo largo de la actividad.

En su encabezado, además del título del formato, tiene aparatados para anotar el número consecutivo del formato, nombre, código y ubicación del equipo. Su cuerpo es una cuadrícula dónde su primera columna indica el mes del año, las siguientes cuatro columnas el número de semana del mes y una sexta columna para observaciones.

En cada recuadro de semana se anotan los correspondientes al tipo de intervención o mantenimiento que se le haya aplicado al equipo, los cuales pueden ser, por ejemplo: L) Lubricación, A) Aseo, R) Reparación, I) Inspección, entre otros. Por último, en la sección inferior del formato, se encuentran tres recuadros de texto.

Del lado izquierdo se escribe el nombre y firma de la persona que realiza la actividad, en el centro el nombre y firma del revisor de la actividad y del lado derecho el nombre y firma de la instancia aprobatoria, que es a quien se reporta la realización del formato. 


\section{Conclusiones}

Con la realización de la presente investigación, se pudieron proponer acciones de mejora mediante la identificación de los indicadores de rendimiento, productividad y eficiencia de los productos bajo estudio, con lo cual se pudo concluir con el logro del objetivo, el cual requería de la identificación de fuentes de desperdicio para la propuesta de mejoras. En este caso, las propuestas que ayudarían a disminuir los desperdicios presentes en el proceso fueron: el programa para el monitoreo de la producción, adaptar la línea de producción a modo de celda de manufactura y la creación de un formato para el mantenimiento preventivo de la maquinaria y equipo.

La naturaleza del proyecto, el cual fue un proyecto de mejora con bases en Lean Manufacturing, no tuvo limitantes de aplicación, ya que, la organización bajo estudio se encuentra en el giro alimenticio, en donde es poco frecuente trabajar con conceptos de manufactura esbelta, por lo que quedó evidenciado que cualquier empresa puede ser diagnosticada bajo este enfoque, independientemente de su giro productivo.

La adopción de una filosofía Lean permite a las empresas un uso más eficiente de los recursos de los que dispone, impactando en cada una de las áreas de la empresa, así como también en los factores externos a ella, como lo son los clientes y los proveedores. Un ejemplo fehaciente es el desarrollo de un VSM, una herramienta Lean que permite el mapeo de procesos, en favor de la detección de actividades que no agregan valor, desde cada una de las áreas o actividades que componen el proceso, hasta aquellas entidades que desempeñan el papel de cliente y proveedor.

Se puede decir que la razón de existir de toda empresa es la generación de bienes monetarios, por lo que una exhaustiva búsqueda de desperdicios en sus procesos permitirá alcanzar con mayor facilidad sus objetivos económicos, volviéndola en una empresa competente, que agrega valor a su entorno. De hacer caso omiso de los hallazgos aquí encontrados en el proceso, las pérdidas económicas generadas por el desperdicio de materias primas, que rondan en torno a los 200 mil pesos, seguirán aquejando al área de rebanado.
La implementación de las tres propuestas en conjunto, no representará costos a la organización, debido en gran medida a que no requiere de una inversión en herramienta o equipo nuevo, sino que las mejoras consisten en la generación de formatos en programas con los que ya dispone la organización, así como una reorganización de la plantilla de trabajo con la que ya cuenta la empresa.

\section{Referencias}

Abdú, H. (2013). La planeación y desarrollo de productos. Bogotá: Universidad Piloto de Colombia.

Álvarez, A. (2014). La medición de la eficiencia y la productividad. En A. Á. Pinilla, La medición de la eficiencia y la productividad (págs. 19-38). Madrid: Prirámide.

Benítez, M. (2012). Análisis y mejora de los Procedimientos de una Empresa de Ingeniería Eléctrica. Obtenido de Biblioteca de Ingeniería Universidad de Sevilla: http://bibing.us.es/proyectos/abreproy/30176/fic hero/03_Capitulo+3\%252FIntroduccion+al+Le an+Manufacturing.pdf

Carro, R., y Gonzalez, D. (2012). Productividad y Competitividad. Obtenido de Nülan: http://nulan.mdp.edu.ar/1607/1/02_productivida d_competitividad.pdf

Corredor, I. (Agosto de 2015). Universidad Nacional Autónoma de México. Obtenido de Repositorio digital de la Facultad de Ingeniería UNAM:

http://www.ptolomeo.unam.mx:8080/xmlui/bits tream/handle/132.248.52.100/7710/Tesis.pdf

División de comercio y Mercados de la FAO. (2018). Perspectivas Alimentarias Noviembre 2018. Ettore Vecchione.

Egas, D. (2017). Proyecto de disminución de desperdicios en el proceso productivo de las máquinas generadoras en la planta Proquinal, S.A. Colombia. Bogotá D.C: Universidad Distrital Francisco José de Caldas.

FIRA. (2016). Panorama Agroalimentario Carne de Cerdo 2016. Obtenido de Gobierno de México:

https://www.gob.mx/cms/uploads/attachment/fi le/200634/Panorama_Agroalimentario_Carne_d e_Cerdo_2016.pdf

CONANT-PABLOS, Marco Antonio, FORNÉS-RIVERA, René Daniel, CANO-CARRASCO, Adolfo y SÁNCHEZ-OSUNA, Adrián. Propuestas para la mejora continua del área de producción de una empresa productora de carne de cerdo, mediante herramientas de manufactura esbelta. Revista de Operaciones Tecnológicas. 2019 
Freivalds, A. y Niebel, B. W. (2014). Ingeniería industrial de Niebel. Métodos, estándares y diseño del trabajo. México: Mc Graw Hill Educación.

Gaucín, D. (11 de Febrero de 2019). Carne de cerdo, un sector con perspectivas de expansión. Obtenido de El economista: https://www.eleconomista.com.mx/opinion/Car ne-de-cerdo-un-sector-con-perspectivas-deexpansion-I-20190211-0094.html

Giannasi, E. (Octubre de 2012). Unión Industrial de Córdoba. Obtenido de Desperdicios en la producción:

http://www.uic.org.ar/Archivos/Revista/File/De sperdicios\%20de\%20la\%20producci\%C3\%B3n -\%20Ef.\%20Em..pdf

Gisbert, J. F. (03 de Septiembre de 2018). Procesos eficientes de negocio como base de la rentabilidad. Obtenido de Inforges: https://www.inforges.es/post/procesos-denegocio-eficientes-como-base-de-larentabilidad

Gobierno del estado de Sonora. (22 de Enero de 2018). Prevén porcicultores sonoreses exportar más en 2018. Recuperado el 9 de Febrero de 2018 , de https://www.sonora.gob.mx/index.php?option= com_content\&view=article\&id=3873:prev\%C3 $\%$ A9n-porcicultores-sonorenses-exportarm\%C3\%A1s-en2018\&catid $=84$ : noticias $\&$ Itemid $=1048$

Hernández, G., y Parrish, M. R. (2017). Mexico's Livestock Sector Remains Healthy. Ciudad de México: Global Agricultural Information Network.

Hernández, J. C., \& Vizán, A. (2013). Lean Manufacturing. Conceptos, técnicas e implantación. Madrid: Fundación EOI.

Jiménez, J., \& Gisbert, V. (22 de Diciembre de 2017). 3 Ciencias . Obtenido de Guía Metodológica de la Gestión de Desperdicios en una PyMe: https://www.3ciencias.com/wpcontent/uploads/2018/01/art_7.pdf
Lobato, M., Gonzalez, Y., \& Teresa, G. (17 de Abril de 2018). Carne de cerdo, retos y oportunidades en la exportación. Obtenido de El economista:

https://www.eleconomista.com.mx/opinion/Car ne-de-cerdo-retos-y-oportunidades-en-laexportacion-I-20180417-0107.html

Madariaga, F. (2013). Lean Manufacturing. Madrid: Bubok Pubishing S.L.

Monteverde, A. (9 de Diciembre de 2018). Porcicultores podrían finalizar año en números rojos. Obtenido de Uniradio noticias: https://www.uniradionoticias.com/noticias/cdob regon/549624/porcicultores-podrian-finalizarano-en-numeros-rojos.html

Montoyo, A. (2011). Universidad de Alicante. Obtenido de Repositorio Institucional de la Universidad de Alicante: https://rua.ua.es/dspace/bitstream/10045/19047/ 1/Tema_4_-_Proceso_de_produccion.pdf

Empresa. (20 de Febrero de 2019a). Entrevista con responsable del proyecto. Obregón, Sonora, México.

Empresa. (22 de Febrero de 2019b). Principales indicadores de desempeño. Obregón, Sonora, México.

Quezada, J. E. (Octubre de 2016). Repositorio usac. Obtenido de Usac (Universidad de San Carlos de Guatemala): http://www.repositorio.usac.edu.gt/5829/1/Josu \%C3\%A9\%20Eduardo\%20Quezada\%20Palaci os.pdf

Redacción. (15 de Noviembre de 2018). Importaciones desmedidas afectan porcicultura mexicana. Ciudad Obregón, Sonora, México.

Redacción Entorno. (26 de Diciembre de 2018). Se consolida Sonora en exportación de carne de cerdo. Obtenido de Entorno Informativo: http://www.entornoinformativo.com.mx/2018/1 2/26/se-consolida-sonora-en-exportacion-decarne-de-cerdo/

Rojas, A., \& Gisbert, V. (2017). Lean Manufacturing: Herramienta para Mejorar la Productividad en las Empresas. Alcoy Alicante, España: 3C Empresa. Investigación y pensamiento crítico. 
Ruíz, J. (2016). Implementación de la Metodología Lean Manufacturing a una Cadena de Producción Agroalimentaria. Sevilla: Universidad de Sevilla.

SAGARPA. (15 de Marzo de 2017). Boletin de prensa. Obtenido de http://www.sagarpa.gob.mx/Delegaciones/jalisc o/boletines/2017/marzo/Documents/2017B0301 5.PDF

Sánchez, R., y Baldenegro, C. (2018). VSM: Aplicación en la reducción de desperdicios en una fábrica de detergentes. Obregón : ITSON.

Sarria, M., Fonseca, G., \& Bocanegra, C. (12 de Junio de 2017). Modelo metodológico de implementación de Lean Manufacturing. Bogotá, Cundinamarca, Colombia .

Sigaudo, D., y Terré, E. (5 de Octubre de 2018). Mercado mundial de cerdos: Argentina en el puesto $13^{\circ}$ de los productores y consumidores. Rosario, Santa Fe, Argentina.

Socconini, L. (2017). Lean Manufacturing, Paso a paso. Guadalajara: Grupo Editorial Norma.

Suñé, A. (2010). Manual práctico de diseño de sistemas productivos. Madrid: Díaz de Santos. 